\title{
HD 144548: A young triply eclipsing system in the Upper Scorpius OB association $\star, \star \star$
}

\author{
R. Alonso ${ }^{1,2}$, H. J. Deeg ${ }^{1,2}$, S. Hoyer ${ }^{1,2}$, N. Lodieu ${ }^{1,2}$, E. Palle ${ }^{1,2}$, and R. Sanchis-Ojeda ${ }^{3,4}$ \\ 1 Instituto de Astrofísica de Canarias, 38205 La Laguna, Tenerife, Spain \\ e-mail: ras@iac.es \\ 2 Dpto. de Astrofísica, Universidad de La Laguna, 38206 La Laguna, Tenerife, Spain \\ 3 Department of Astronomy, University of California, Berkeley, CA 94720, USA \\ 4 NASA Sagan Fellow
}

Received 3 August 2015 / Accepted 14 October 2015

\section{ABSTRACT}

\begin{abstract}
The star HD 144548 (=HIP 78977; TYP 6212-1273-1) has been known as a detached eclipsing binary and a bona-fide member of the Upper Scorpius OB association. Continuous photometry from the K2 mission on Campaign Two has revealed the presence of additional eclipses due to the presence of a third star in the system. These are explained by a system composed of the two previously known members of the eclipsing system $(B a$ and $B b)$ with a period of 1.63 d, orbiting around an F7-F8V star with a period of $33.945 \pm 0.002 \mathrm{~d}$ in an eccentric orbit $\left(e_{A}=0.2652 \pm 0.0003\right)$. The timing of the eclipses of $B a$ and $B b$ reveals the same $33.9 \mathrm{~d}$ periodicity, which we interpret as the combination of a light time effect combined with dynamical perturbations on the close system. Here we combine radial velocities and analytical approximations for the timing of the eclipses to derive masses and radii for the three components of the system. We obtain a mass of $1.44 \pm 0.04 M_{\odot}$ and radius of $2.41 \pm 0.03 R_{\odot}$ for the $A$ component, and almost identical masses and radii of about $0.96 M_{\odot}$ and $1.33 R_{\odot}$ for each of the two components of the close binary. HD 144548 is the first triply eclipsing system for which radial velocities of all components could be measured.
\end{abstract}

Key words. binaries: eclipsing - techniques: photometric

\section{Introduction}

Triply eclipsing stars are very rare systems, in which all components of a triple hierarchical stellar system are mutually eclipsing. When the three stars are in a compact configuration, they are excellent laboratories to check dynamical effects that have shorter timescales than in stellar binaries, and from which information on the stellar interiors can be inferred (e.g. Claret \& Gimenez 1993). If the system is observed photometrically during a long period of time, it is possible to measure the physical parameters of the three stars with remarkable accuracy. KOI-126 was the first triply eclipsing system found (Carter et al. 2011), and it allowed the determination of the masses and radii for two of the components down to $3 \%$ and $0.5 \%$ fractional uncertainty, respectively, with a combination of a photo-dynamical model and radial velocity observations. Two other triply eclipsing systems have been found thanks to the Kepler mission (HD 181068, Derekas et al. 2011; KIC 002856960, Armstrong et al. 2012; Lee et al. 2013), while 26 other triple systems (the majority not showing eclipses of the outer star) have been detected thanks to the study of the eclipse time variations (Rappaport et al. 2013; Borkovits et al. 2015).

^ Partially based on observations made with the Italian Telescopio Nazionale Galileo (TNG) operated by the Fundación Galileo Galilei of the INAF, the Nordic Optical Telescope, operated by the Nordic Optical Telescope Scientific Association, and the William Herschel Telescope (programme DDT58 - PI Lodieu) operated by the Isaac Newton Group on the island of La Palma at the Spanish Observatorio Roque de los Muchachos of the IAC. This paper includes data collected by the Kepler mission. Funding for the Kepler mission is provided by the NASA Science Mission directorate.

$\star \star$ Appendices are available in electronic form at http://www . aanda.org
Upper Scorpius (USco) is part of the nearest OB association $(145 \mathrm{pc})$. The central region of USco is free of extinction and star formation has already ended (Walter et al. 1994). The age of USco remains under debate. Preibisch \& Zinnecker (1999) originally estimated an age of 5-6 Myr with a small dispersion based on the location of pre-main-sequence members in the HR diagram. Recently, Pecaut et al. (2012) revised the age of the isochronal ages of intermediate-mass members to $11 \pm 2 \mathrm{Myr}$ in contrast to the $4 \pm 1 \mathrm{Myr}$ age derived for low-mass members (Slesnick et al. 2008; Lodieu et al. 2011; Herczeg \& Hillenbrand 2015). For HD 144548, most models suggest an age of 8-11 Myr, its kinematic distance is $133 \pm 12 \mathrm{pc}$, and it is classified as a F8V star in USco with an effective temperature of $6138 \mathrm{~K}$ and an $A_{v}$ of $0.38 \pm 0.08 \mathrm{mag}$ (Table 4 of Pecaut et al. 2012). HD 144548 is an astrometric member of USco based on HIPPARCos data (de Zeeuw et al. 1999; Pecaut et al. 2012), with reported detection of lithium and $24 \mu \mathrm{m}$ excess due to a debris disk (Chen et al. 2011), and it is a known X-ray source from ROSAT data (Haakonsen \& Rutledge 2009). HD 144548 had been identified by Kiraga (2012) as a detached eclipsing binary system with ASAS photometry of ROSAT sources. These authors reported a period of $0.81292 \mathrm{~d}$, although they note a possible period twice as long. In this work, we identify a period of $1.63 \mathrm{~d}$ for the orbit of a close binary system, further denoted binary 1 with components $B a$ and $B b$, which is itself orbiting around a longer period (33.9 d) eclipsing component (binary 2, component $A$ ), forming a compact hierarchical, triply eclipsing system $^{1}$.

\footnotetext{
1 After acceptance of the manuscript, we became aware of an independent work by Ripepi et al. (2015) in which they pointed out the potential hierarchical triple nature of the system.
} 


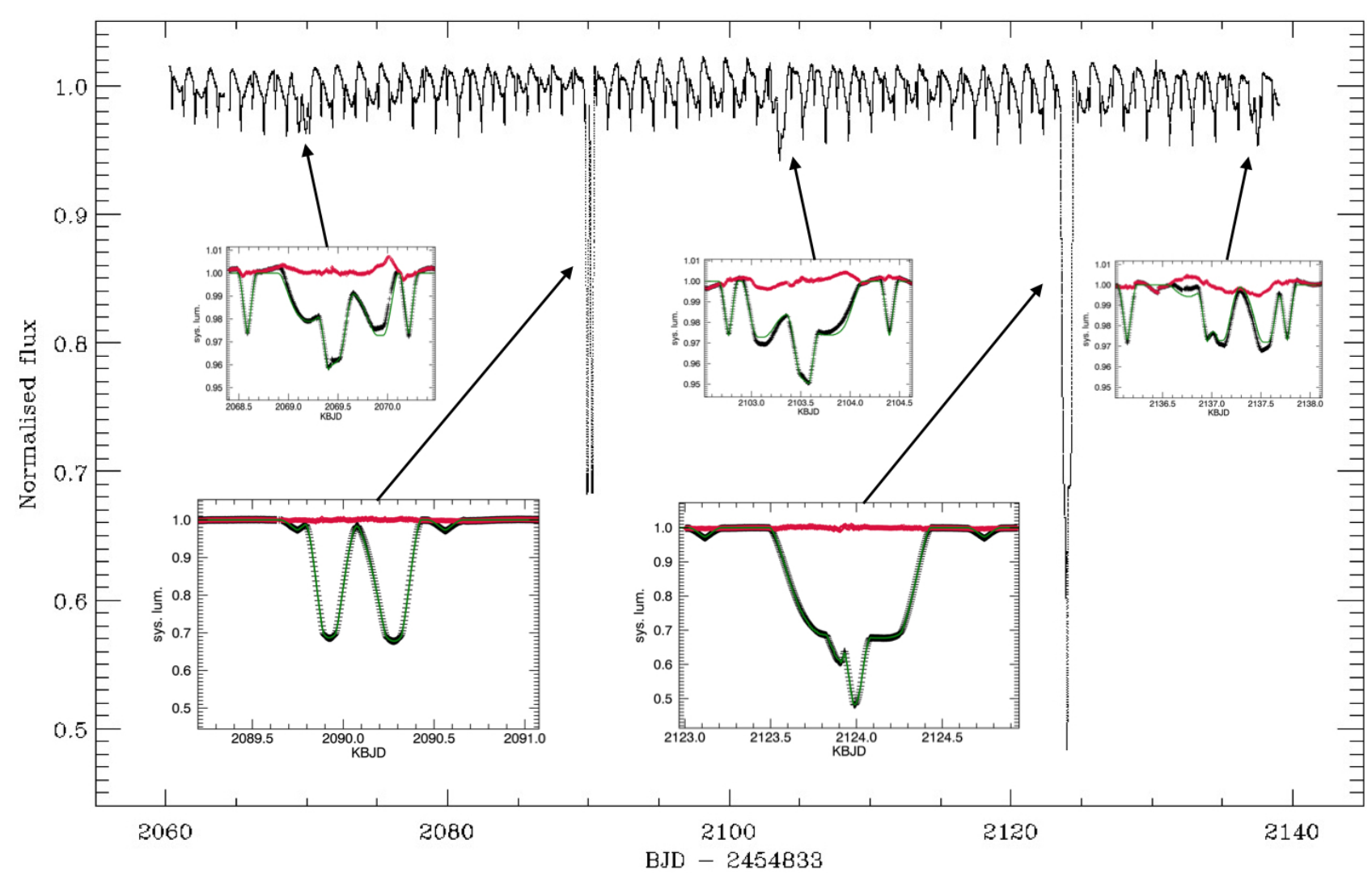

Fig. 1. Extracted K2 light curve of HD 144548, showing periodic eclipses of a hierarchical triple eclipsing system and clear modulation, which we attribute to the effect of rotation and spots. The insets show primary and secondary eclipses of $A$ vs. $B_{a}$ and $B_{b}$ components, with the best-fit model (green dotted lines) and residuals of the fit (red crosses, shifted vertically to the off-eclipse flux).

\section{Observations and data analysis}

The K2 (Howell et al. 2014) Campaign Two data ${ }^{2}$ for the star HD 144548 (EPIC-204506777) spans $78.78 \mathrm{~d}$, starting on Aug. 23, 2014. The star is bright $(V=8.75$, Kepmag $=8.75)$ and appears slightly saturated in the target pixel files.

We extracted the photometry from the short cadence data, which consists of 115680 windows with a size of $16 \times 29$ pixels approximately centred on the target star. For each time stamp, we determined the centroid by performing Gaussian fits to the mean flux level at each spatial direction to avoid two-dimensional fits that may get confused by the changing vertical trails arising from the saturation of the images. A circular aperture with a radius of 7 pixels was used to obtain the flux from the target. The columns close to the determined $\mathrm{X}$-centre of the target were summed in their whole length to include the flux that was dispersed as a result of saturation. The background level was estimated from an 8.4 pixel-wide annulus starting 9.1 pixels away from the measured centroid. Several outliers due to cosmic rays impacts were located and interpolated through a 5-sigma clipping moving median window. Less than $0.8 \%$ of the data points were corrected this way. The final light curve is plotted in Fig. 1, where two deep eclipses of the close binary passing before component A are dominant in the curve; these eclipses are about 34 days apart. We also detected three occultations of the binary star behind the star $A$. The mutual eclipses of the $B a-B b$ system are seen throughout the whole light curve with a period of $1.6278 \mathrm{~d}$.

\footnotetext{
2 Proposed for observations in long cadence mode ( $\sim 30$ min cadence) by Guest Observer programmes 2020, 2021, 2023, 2034, 2049, 2052, 2086, 2092, and in short cadence mode ( $\sim 1$ min cadence) by programme 2023.
}

The light curve exhibits a modulation of a few percent between eclipses of the close components. The autocorrelation function of this modulation shows a maximum at a period close to, but not exactly, the orbital period of the close binary 1 . A division of the curve used to compute the autocorrelation by a 20 points median-smoothed version is used to estimated the dispersion of the residuals as $210 \mathrm{ppm}$.

\subsection{Observed minus calculated $(\mathrm{O}-\mathrm{C})$ diagram}

The highly significant individual eclipses of close binary 1 allow for a timing of their centre positions. The timing of the eclipses departs from a strict periodicity due to a combination of the light travel time effect (LTTE) and several dynamical effects that change the intrinsic period of binary 1 . A complete description of the dynamical effects is found in Borkovits et al. (2015), hereafter B15. To describe the $\mathrm{O}-\mathrm{C}$ diagram, we implemented the analytical equations of that work, including the following terms: i) the LTTE, and ii) dynamical effects with a timescale of the outer binary period up to second order $\left(\Delta_{1}\right.$, Eq. (5) of (B15), and $\Delta_{2}$, Appendix A of (B15)). We added a linear trend in the argument of periastron $\dot{\omega}_{2}$ to account for a possible apsidal rotation.

The preparation of the light curve and the extraction of the $\mathrm{O}-\mathrm{C}$ diagram is described in further detail in Appendix A. The result is plotted in Fig. 2. We performed an initial fit of the $\mathrm{O}-\mathrm{C}$ times using the AMOEBA minimization algorithm (Press et al. 1992), which included 18 parameters to the fit. As the number of data points is comparable to the model parameters, the fit is unconstrained and at this stage it was only used to prepare the data for the final modelling described in Sect. 2.3. 


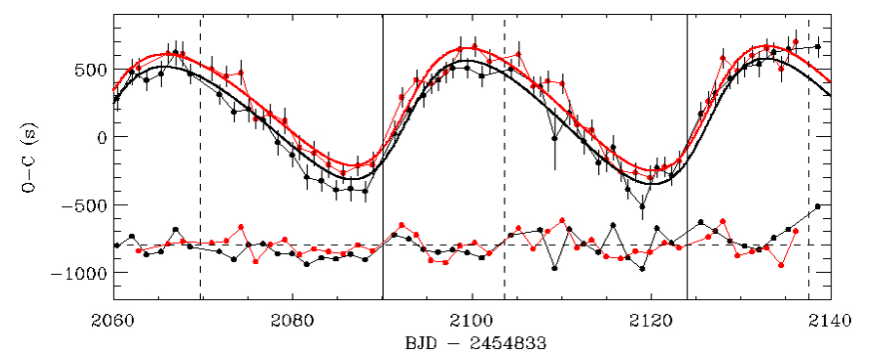

Fig. 2. Observed minus calculated diagram, and best-fit model. Red: primary eclipses; black: secondary eclipses. The vertical lines mark the positions of the eclipses and occultations of the outer binary 2 .

\subsection{Radial velocities}

While a fit to the $\mathrm{O}-\mathrm{C}$ in the previous section is in principle the equivalent of observing a double-lined spectroscopic binary, an increased precision of the orbital parameters of the system is obtained with radial velocity measurements (Carter et al. 2011). The system HD 144548 was observed at the $2.5 \mathrm{~m}$ NOT with the FIES spectrograph (Telting et al. 2014) on the night of May 7th, 2015. The exposure time was $1800 \mathrm{~s}$. Four additional measurements were obtained with the HARPS-N spectrograph at the $3.6 \mathrm{~m}$ TNG on four consecutive nights starting on May 21st, 2015, using an exposure time of $900 \mathrm{~s}$ and reaching a signalto-noise ratio in the red part of around 130 per spectrum. An additional spectrum was taken with the ISIS spectrograph at the William Herschel Telescope on June 8th, 2015. For the FIES and ISIS data points, the spectra were reduced and extracted with regular tools (the online data reduction FIESTOOL ${ }^{3}$ and IRAF $^{4}$ ). The radial velocities were obtained with IRAF FXCOR, and standard radial velocity stars observed on the same night were used to obtain the absolute velocities.

For the HARPS-N spectra, we obtained the cross-correlation functions (CCFs) over a $400 \mathrm{~km} \mathrm{~s}^{-1}$ velocity region with the online YABI tool (Borsa et al. 2015; Hunter et al. 2012) with the use of a G2 mask. The CCF is dominated by a broad $\left(\sim 160 \mathrm{~km} \mathrm{~s}^{-1}\right)$ peak and shows signs of an additional CCF component at velocities around $+100 \mathrm{~km} \mathrm{~s}^{-1}$ (Fig. 3). We attribute these secondary peaks to one of the components of the close binary system. With the use of the ephemeris of the three components, and making first reasonable guesses as to the masses of each system component, we estimated the position of the CCF of the second component of the close binary system to lie blended with the right wing of the CCF of the A component of the system. According to this, the first obtained HARPS-N spectrum is the least contaminated by this component, and we used its velocity as a reference point. For the other HARPS-N spectra, we estimated the radial velocity shifts via a cross-correlation of the $\mathrm{CCF}$ using only the left wing of the reference CCF. For the secondary CCFs visible around $100 \mathrm{~km} \mathrm{~s}^{-1}$, we estimated their central position by a baseline normalization and a fit to a Gaussian with a $\mathrm{K} 5 \mathrm{~V}$ correlation mask. The estimation of the centre of the secondary CCF using two different functions (Gaussian and a trapezoid) was used to evaluate the error bars, which are summarized in Table B.1. The best orbital fit to the three components of the system is plotted in Fig. 3. To perform this fit, we limited the rate of apsidal motion $\dot{\omega}_{2}$ of binary 2 to the values obtained from the light-curve fitting described in the following section, as this quantity is better constrained by the ephemeris of the primary and secondary eclipses of the $A$ component.

\footnotetext{
3 http://www .not.iac.es/instruments/fies/fiestool/

4 http://iraf.noao.edu/
}

\subsection{Light curve modelling of the eclipses}

Fits of the stellar photometric eclipses were obtained with a revised version of the UFIT fitter programme using the AMOEBA algorithm against models generated with the Universal Transit Modeller (UTM; both Deeg 2014). The UTM permits the generation of light curves from any number of mutually occulting objects (stars, planets, moons, planetary rings) on arbitrary elliptical orbits in hierarchical systems. The bodies' orbital positions are based on the usual elliptical orbital parameters: period $P$, semimajor axis $a$, eccentricity $e$, argument of periapsis $\omega$, inclination $i$, longitude of ascending node (or position angle) $\Omega$, time relative to a reference epoch, and apsidal motions $\dot{\omega}$.

In a first fitting pass, we used the average phased curves of binary 1 , shown in Fig. A.2, to derive this binary's relative radii $R_{B a} / a_{1}$ and $R_{B b} / a_{1}$, radius ratio $k_{1}=R_{B b} / R_{B a}$, luminosity ratio $q_{L 1}=L_{B b} / L_{B a}$, inclination $i_{1}$, and the fraction of its luminosity relative to the total luminosity, with all other light assumed to be from the component $A$. For the limb darkening, we coupled the coefficients of the two very similar components and fitted only a linear coefficient, since neither individual coefficients nor higher-order limb darkening resulted in significantly better fits. The residuals of these fits to binary 1 , of 0.0031 in normalized flux units, are dominated by asymmetries in their eclipse events, which are likely due to inhomogeneous surfaces of these stars.

In the second pass, we concentrated on the eclipses involving component $A$. For these fits, a light curve was used in which each time stamp was corrected for the $\mathrm{O}-\mathrm{C}$ time resulting from the fit to $\mathrm{O}-\mathrm{C}$ times described in Sect. 2.1. Thus, the shallow eclipses of the binary 1 appear with strict periodicity. The light curve was also median-filtered and rebinned by a factor of 5 . Furthermore, we trimmed the light curve to include only sections around the $A$-component eclipses, extended to include one binary- 1 eclipse on either side. Parameters derived from the fit to binary 1 were initially kept fixed, although we permitted small variations in the final fits. Linear limb-darkening parameters were used. As expected, the eccentricity $e_{2}$ and argument of periapsis $\omega_{2}$ were strongly correlated. We proceeded therefore with fits in which we gave as initial conditions an estimate of the phase $\phi_{s, 2}$ of secondary eclipses on component $A$ and for a given value $\omega_{2}$ (that was kept fixed), calculated an initial eccentricity $e_{2}$. Fits were then repeated for sets of fixed values of $\omega_{2}$.

\subsection{Combined fit}

We used the obtained orbital inclinations, periods, phase of secondary eclipse of component $A$, and apsidal motion to perform a combined fit of the radial velocity and $\mathrm{O}-\mathrm{C}$ curves, using these as priors. In the fits, we also changed the initial values of the remaining parameters slightly and we computed 4000 AMOEBA chains, which converged after $\sim 2000$ iterations per chain. The distributions of all the iterations with a $\chi^{2}$ lower than the final $\chi^{2}$ of the worst converging chain were used to estimate the final error bars.

The best light-curve fit, for which the values in Table B.2 are given, has an overall residual rms of 0.0021 in normalized flux units within the main eclipses involving component A (insets of Fig. 1), and 0.0017 for the rest of the light curve. These residuals are an order of magnitude smaller than the $\sim 3 \%$ peak-topeak variability of the raw light curve (Fig. 1). The major origin of the residuals is probably an imperfect removal of that variability, which is likely caused by surface inhomogeneities of the stellar surfaces on any of the components. During eclipse events, 

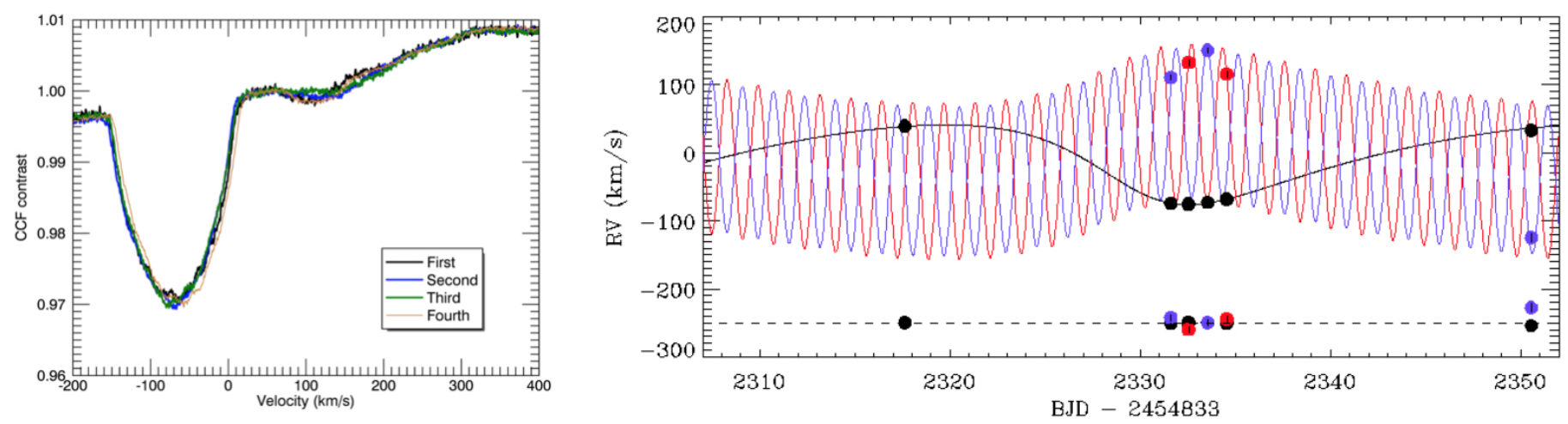

Fig. 3. Left: HARPS-N CCFs obtained on four consecutive nights, showing a wide main component at about $-80 \mathrm{~km} \mathrm{~s}^{-1}$, and more subtle CCFs around $100 \mathrm{~km} \mathrm{~s}^{-1}$. Right: best-fit orbital solution for the three components of the system (black: $A$; blue: $B a$; red: $B b$ ), and the residuals of the fit shifted for clarity (points near dashed line). These are $\sim 1.6 \mathrm{~km} \mathrm{~s}^{-1}$ for the $A$ component and $\sim 11 \mathrm{~km} \mathrm{~s}^{-1}$ for each of the components of binary 1 .

these inhomogeneities may cause further brightness variations on time-scales that are different than the off-eclipse variations, and which cannot be corrected for. Another source of deviation comes from limits in the model-generation, which is based on Keplerian orbits plus apsidal motion. The dominant source of the observed $\mathrm{O}-\mathrm{C}$ residuals are dynamical effects on shorter timescales, which ultimately arise from deviations of the bodies' orbits from Keplerian orbits. These deviations against the modelled positions of the bodies may of course affect the modelled eclipse light curves. A full dynamical three-body simulation of the system, as was done with KOI-126 (Carter et al. 2011), or a conversion of the analytical terms describing the $\mathrm{O}-\mathrm{C}$ deviations in the framework of B15 into geometric deviations might remedy this. This is however beyond the scope of this work and given the rather large flux variations of the system not arising from eclipses, it might not lead to a relevant improvement in the knowledge of the system's parameters.

\section{Conclusions}

We report the discovery of a young (8-11 Myr) triply eclipsing system member of the nearest OB association to the Sun. The physical parameters obtained for the each of the components of the system are good calibrators for stellar models at young ages. The obtained radii of the stars are about $65 \%$ and $40 \%$ larger than main-sequence stars for the component $A$ and each of the components $B a-B b$, respectively, as is expected because of the young age of the system. We emphasize that HD 144548 is the youngest and one of the most compact triple systems known, the other being KOI-126 (outer period $33.92 \mathrm{~d}$ ) and $\lambda$-Tau (outer period $33.03 \mathrm{~d}$, Tokovinin 2008). This accumulation near $P_{2} \sim 33 \mathrm{~d}$ might hint at dynamical limits in the origin of these compact triple systems. From the difference between inclinations $\left(i_{1}-i_{2}\right)$ and from $\triangle \mathrm{PA}$, we conclude that the orbital planes are coplanar within $2 \mathrm{deg}$.

Given the brightness of the system, we expect that further observations of eclipses of component $A$ will be a rewarding topic even for observatories with modest equipment. Additional radial velocities at $\sim \mathrm{km} \mathrm{s}^{-1}$ precision might also help to calibrate some possible remaining systematics, such as the effect of the stellar activity or the extraction of the RVs for the close binary 1 , and refine the parameters, especially the dynamical effects, described in this work. Finally, a determination of the spinorbit alignment of the component $A$ vs. component $B a-B b$ and between both components of the close system from observations of the Rossiter/McLaughlin effect would provide constraints to tidal forces on young stars.

Acknowledgements. We thank the anonymous referee for useful comments that helped to improve the paper. R.A. and N.L. were funded by the Ramón y Cajal fellowship (RYC-2010-06519 and 08-303-01-02, respectively). H.D. and S.H. acknowledge support by grant AYA2012-39346-C02-02 and under the 2011 Severo Ochoa Programme SEV-2011-0187, both from the Spanish Secretary of State for R\&D\&i (MINECO). R.A., E.P. acknowledge funding from MINECO grants ESP2013-48391-C4-2-R and ESP2014-57495-C2-1-R. RSO acknowledges NASA/JPL funding through the Sagan Fellowship Program executed by the NASA Exoplanet Science Institute. We thank the WHT staff and its service programme, the TNG staff, the NOT staff, and Jorge P. Arranz for help during the observations.

\section{References}

Armstrong, D., Pollacco, D., Watson, C. A., et al. 2012, A\&A, 545, L4 Borkovits, T., Rappaport, S., Hajdu, T., \& Sztakovics, J. 2015, MNRAS, 448, 946

Borsa, F., Scandariato, G., Rainer, M., et al. 2015, A\&A, 578, A64

Carter, J. A., Fabrycky, D. C., Ragozzine, D., et al. 2011, Science, 331, 562

Chen, C. H., Mamajek, E. E., Bitner, M. A., et al. 2011, ApJ, 738, 122

Claret, A., \& Gimenez, A. 1993, A\&A, 277, 487

de Zeeuw, P. T., Hoogerwerf, R., de Bruijne, J. H. J., Brown, A. G. A., \& Blaauw, A. 1999, AJ, 117, 354

Deeg, H. J. 2014, UTM: Universal Transit Modeller, Astrophys. Source Code Libr. [recordascl: 1412.003]

Derekas, A., Kiss, L. L., Borkovits, T., et al. 2011, Science, 332, 216

Haakonsen, C. B., \& Rutledge, R. E. 2009, ApJS, 184, 138

Herczeg, G. J., \& Hillenbrand, L. A. 2015, ApJ, 808, 23

Howell, S. B., Sobeck, C., Haas, M., et al. 2014, PASP, 126, 398

Hunter, A. A., Macgregor, A. B., Szabo, T. O., Wellington, C. A., \& Bellgard, M. I. 2012, Source Code Biol Med, 7, 1

Kiraga, M. 2012, Acta Astron., 62, 67

Lee, J. W., Kim, S.-L., Lee, C.-U., et al. 2013, ApJ, 763, 74

Lenz, P., \& Breger, M. 2005, Commun. Asteroseismol., 146, 53

Lodieu, N., Dobbie, P. D., \& Hambly, N. C. 2011, A\&A, 527, A24

Pecaut, M. J., Mamajek, E. E., \& Bubar, E. J. 2012, ApJ, 746, 154

Preibisch, T., \& Zinnecker, H. 1999, AJ, 117, 2381

Press, W. H., Teukolsky, S. A., Vetterling, W. T., \& Flannery, B. P. 1992, Numerical recipes in FORTRAN, The art of scientific computing 2nd edn. (Cambridge: University Press)

Rappaport, S., Deck, K., Levine, A., et al. 2013, ApJ, 768, 33

Ripepi, V., Balona, L., Catanzaro, G., et al. 2015, MNRAS, 454, 2606

Slesnick, C. L., Hillenbrand, L. A., \& Carpenter, J. M. 2008, ApJ, 688, 377

Telting, J. H., Avila, G., Buchhave, L., et al. 2014, Astron. Nachr., 335, 41

Tokovinin, A. 2008, MNRAS, 389, 925

Walter, F. M., Vrba, F. J., Mathieu, R. D., Brown, A., \& Myers, P. C. 1994, AJ, 107,692

Pages 5 to 6 are available in the electronic edition of the journal at http://www . aanda.org 


\section{Appendix A: Obtaining the $\mathrm{O}-\mathrm{C}$ diagram}

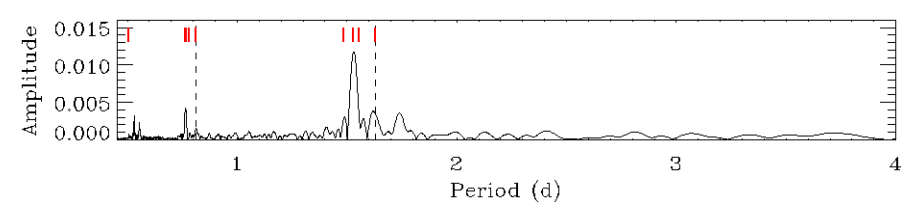

Fig. A.1. Amplitude periodogram of the light curve shown with all the eclipses removed and interpolated. The vertical dashed lines indicate the period of the close binary 1 and its harmonics. The red ticks indicate the position of the frequencies removed for further analysis.
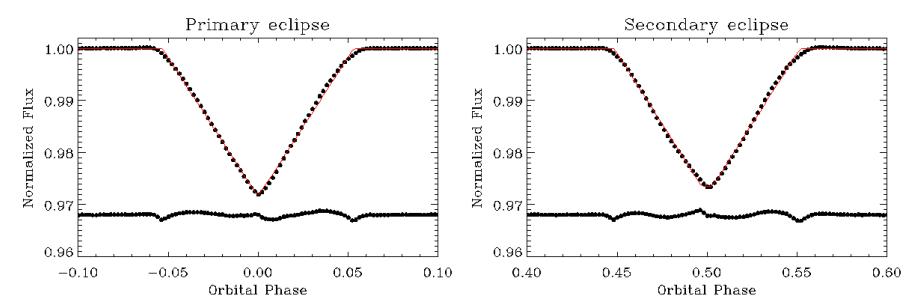

Fig. A.2. Averaged curves of the primary and secondary eclipses of close binary 1 and best-fit trapezoid model to each of them. The points around 0.968 are the residuals, shifted vertically for visualization. There are significant differences from the simple trapezoid model, but as these are approximately symmetrical from the centre of the eclipses, they are not expect to introduce systematic errors in the determination of the time of eclipse centres.

To prepare the light curve for the analysis, first we cleaned the most significant frequencies in the amplitude spectrum of the off-eclipse sections, using PERIOD04 (Lenz \& Breger 2005). Twelve sinusoidal components were used, until amplitudes of the peaks of about three times the dispersion of the residuals were reached. Figure A.1 shows the amplitude spectrum and the frequencies that were removed. As in the case of the autocorrelation, the spectrum shows frequencies that are close to, but not exactly, that of binary 1; the spectrum also shows peaks at the orbital frequency of the binary 1 and its harmonics. These might be caused by a combination of reflected light, ellipsoidal variability, and doppler beaming whose detailed analysis is beyond the scope of this paper.

We used a parabolic fit to the baseline to remove the modulation between the eclipses, and a Levenberg-Marquardt fit (Press et al. 1992) to a trapezoidal function to estimate the parameters of each eclipse (centre, depth, duration, and ingress time). Of the four fit parameters for each eclipse, only the central time of the eclipse shows a significant variability, while the other three remain constant. The observed variability has the same period as the wide component $A$. On a final iteration, we corrected for the measured O-C displacement at each time stamp before computing the average curves for both the primary and secondary eclipse of the inner binary (templates), which are plotted in Fig. A.2. For each observed eclipse, the corresponding template was shifted in time on a regular grid and the $\chi^{2}$ between the model and the data was calculated and used to calculate the time of eclipse centre and its uncertainty.

\section{Appendix B: Additional tables}

Table B.1. Radial velocities.

\begin{tabular}{lcrrr}
\hline \hline $\begin{array}{l}\text { BJD - } \\
2450000\end{array}$ & Source & $\begin{array}{r}\mathrm{RV}_{A} \\
{\left[\mathrm{~km} \mathrm{~s}^{-1}\right]}\end{array}$ & $\begin{array}{r}\mathrm{RV}_{B a} \\
{\left[\mathrm{~km} \mathrm{~s}^{-1}\right]}\end{array}$ & $\begin{array}{r}\mathrm{RV}_{B b} \\
{\left[\mathrm{~km} \mathrm{~s}^{-1}\right]}\end{array}$ \\
\hline 7150.63672 & FIES & $39.1 \pm 0.4$ & - & - \\
7164.62688 & HARPS-N & $-74.2 \pm 0.2$ & $110.7 \pm 4.0$ & - \\
7165.55411 & HARPS-N & $-75.7 \pm 0.2$ & - & $132.7 \pm 4.0$ \\
7166.56063 & HARPS-N & $-73.0 \pm 0.2$ & $150.3 \pm 4.0$ & - \\
7167.55627 & HARPS-N & $-68.5 \pm 0.2$ & - & $115.7 \pm 4.0$ \\
7183.56465 & ISIS & $32.7 \pm 3.4$ & $-125 \pm 6.0$ & - \\
\hline
\end{tabular}


Table B.2. Parameters of the triple system.

\begin{tabular}{|c|c|}
\hline \multicolumn{2}{|l|}{ Light-curve fit of binary 1 mutual eclipses } \\
\hline $\begin{array}{l}\text { period, } P_{1}(\mathrm{~d}) \\
\text { epoch }^{1}, T_{1}(\mathrm{BKJD})^{2} \\
\text { inclination, } i_{1}(\mathrm{deg}) \\
\text { eccentricity, } e_{1} \\
\text { relative star radius, } R_{B a} / a_{1} \\
\text { radius ratio, } k_{1}=R_{B b} / R_{B a} \\
\text { comp. } B a, B b \text { linear limb-dark. coeff., } u_{a} \\
\text { luminosity ratio, } L_{B b} / L_{B a}\end{array}$ & $\begin{array}{r}1.6278 \pm 0.0001 \\
2061.2610 \pm 0.0012 \\
88.9 \pm 0.5 \\
\lesssim 0.0015 \\
0.1834 \pm 0.0012 \\
1.008 \pm 0.005 \\
0.78 \pm 0.07 \\
0.97 \pm 0.05\end{array}$ \\
\hline \multicolumn{2}{|l|}{ Light-curve fit of binary 2 (eclipses with component $A$ ) } \\
\hline $\begin{array}{l}\text { period, } P_{2}(\mathrm{~d}) \\
\text { epoch }^{3}, T_{2}(\mathrm{BKJD}) \\
\text { inclination, } i_{2}(\mathrm{deg}) \\
\text { relative position angle }, \Delta P . A . \\
\text { secondary eclipse phase }^{4}, \phi_{s, 2} \\
\text { eccentricity, } e_{2} \\
\text { arg. of periapsis }{ }^{6}, \omega_{2}(\mathrm{deg}) \\
\text { apsidial rotation velocity, } \dot{\omega}_{2}\left(\mathrm{deg} \mathrm{d}^{-1}\right) \\
\text { epoch of periapsis, } T_{v, 2}(\mathrm{BKJD}) \\
\text { relative star radius, } R_{A} / a_{2} \\
\text { comp. } A \text { limb-dark. coeff., } u \\
\text { luminosity ratio, }\left(L_{B a}+L_{B b}\right) / L_{A} \\
\text { mass ratio } q_{12}=\left(m_{B a}+m_{B b}\right) / m_{A} \\
\text { mass ratio } q_{1}=m_{B b} / m_{B a}\end{array}$ & $\begin{array}{r}33.945 \pm 0.002 \\
2090.0680 \pm 0.0010 \\
89.28 \pm 0.05 \\
-1.0 \pm 0.9 \\
0.3950 \pm 0.0005 \\
0.2652 \pm 0.0003 \\
127.63 \pm 0.15 \\
0.0235 \pm 0.002 \\
2092.31 \pm 0.02 \\
0.0364 \pm 0.0005 \\
0.37 \pm 0.10 \\
0.0593 \pm 0.0012 \\
1.34 \pm 0.03 \\
0.995 \pm 0.015\end{array}$ \\
\hline \multicolumn{2}{|l|}{ Parameters from $\mathrm{O}-\mathrm{C}$ and $\mathrm{RV}$ fit } \\
\hline $\begin{array}{l}\text { amplitude of light travel time effect, } A_{\mathrm{LTTE}}(\mathrm{s}) \\
\text { amplitude of first order dynamical effects with } \mathrm{P}_{2} \text { timescale, } A_{L 1}(\mathrm{~s}) \\
\text { amplitude of second order dynamical effects with } \mathrm{P}_{2} \text { timescale, } A_{L 2}(\mathrm{~s}) \\
\text { mutual orbital inclination } i_{m}(\text { degrees }) \\
\text { RV semi-amplitude } K_{A}\left(\mathrm{~km} \mathrm{~s}^{-1}\right) \\
\text { RV semi-amplitude } K_{B a+B b}\left(\mathrm{~km} \mathrm{~s}^{-1}\right) \\
\text { RV semi-amplitude } K_{B a}\left(\mathrm{~km} \mathrm{~s}^{-1}\right) \\
\text { RV semi-amplitude } K_{B b}\left(\mathrm{~km} \mathrm{~s}^{-1}\right) \\
\text { RV offset, gamma }\left(\mathrm{km} \mathrm{s}^{-1}\right) \\
\text { mass ratio } q_{12}=\left(m_{B a}+m_{B b}\right) / m_{A} \\
\text { mass ratio } q_{1}=\left(m_{B b} / m_{B a}\right)\end{array}$ & $\begin{array}{r}86.7 \pm 0.1 \\
957 \pm 8 \\
2.4 \pm 0.9 \\
0.2 \pm 0.5 \\
58.52 \pm 0.05 \\
43.6 \pm 0.6 \\
110.2 \pm 1.2 \\
115.0 \pm 0.6 \\
-6.97 \pm 0.10 \\
1.342 \pm 0.019 \\
0.959 \pm 0.015\end{array}$ \\
\hline \multicolumn{2}{|l|}{ Adopted parameters } \\
\hline $\begin{array}{l}\text { semimajor axis, } a_{A}(\mathrm{AU}) \\
\text { semimajor axis, } a_{B a+B b}(\mathrm{AU}) \\
\text { semimajor axis, } a_{B a}(\mathrm{AU}) \\
\text { semimajor axis, } a_{B b}(\mathrm{AU}) \\
\text { semimajor axis, } a_{1}(\mathrm{AU}) \\
\text { semimajor axis, } a_{2}(\mathrm{AU}) \\
\text { mass component } A, M_{A}\left(M_{\odot}\right) \\
\text { mass component } B_{a}, M_{B a}\left(M_{\odot}\right) \\
\text { mass component } B_{b}, M_{B b}\left(M_{\odot}\right) \\
\text { equatorial radius component } A, R_{A}\left(R_{\odot}\right) \\
\text { radius component } B_{a}, R_{B a}\left(R_{\odot}\right) \\
\text { radius component } B_{b}, R_{B b}\left(R_{\odot}\right)\end{array}$ & $\begin{array}{r}0.17619 \pm 0.00012 \\
0.1313 \pm 0.0018 \\
0.0165 \pm 0.0002 \\
0.01721 \pm 0.00009 \\
0.03371 \pm 0.00011 \\
0.3075 \pm 0.0013 \\
1.44 \pm 0.04 \\
0.984 \pm 0.007 \\
0.944 \pm 0.017 \\
2.41 \pm 0.03 \\
1.319 \pm 0.010 \\
1.330 \pm 0.010\end{array}$ \\
\hline
\end{tabular}

Notes. ${ }^{(1)}$ Epoch of the primary (deeper) eclipse, when component $B a$ is being occulted by $B b .{ }^{(2)}$ Barycentric Kepler Julian Date, BKJD $=$ BJD 2454833 . ${ }^{(3)}$ Epoch when component A would become eclipsed by the barycentre of the compact binary 1. ${ }^{(4)}$ Orbital phase of component A when the barycentre of components $B a, B b$ eclipses behind it. ${ }^{(5)}$ Position angle of orbital plane of binary 2 relative to orbital plane of binary $1 .{ }^{(6)}$ This value changes with time due to $\dot{\omega}_{2}$ and is given for the moment of the epoch $T_{v, 2}$. 